\title{
PRESENTATIONS FOR UNIT GROUPS OF MODULAR GROUP ALGEBRAS OF GROUPS OF ORDER 16
}

\author{
ROBERT SANDLING
}

\begin{abstract}
For a $p$-group $G$ and the field $F$ of $p$ elements, let $V$ denote the group of normalized units of the group algebra $F G$. Generators and relations are provided for $V$ for each group $G$ of order dividing 16 . The presentations are sufficiently concise to permit transcription for machine calculation with $V$. Some applications are described. A theoretical method for obtaining presentations for $V$ is developed. It is most effective when $p=2$, or when $p=3$ and the commutator subgroup $G^{\prime}$ is of order 3. Implementation involves extensive calculation in $F G$.
\end{abstract}

\section{INTRODUCTION}

The unit group of the ring of matrices over a field is a well-studied and familiar object, the general linear group. This example is, however, atypical: the structure of the group of units of a ring is usually elusive. Efficient presentations are rarely available. This paper develops techniques for obtaining a useful presentation of the unit group of the modular group algebra $F G$, where $F$ is the field of $p$ elements and $G$ is a finite $p$-group. Explicit presentations, obtained with the additional assistance of machine calculation, are given for some small cases.

A more specific reason for seeking presentations in this context is the traditional one of compiling data for use in formulating conjectures and testing hypotheses. The data gathered here have already shown their value. A condition is established in [16] for the existence of a normal complement to $G$ in the group of units of $F G$. It was motivated by the normal complements found in cases where $|G|=16$ by the use of the presentations of this paper in conjunction with group theory packages (computers had been used earlier $[12,7]$ to study this question but not by means of presentations). In the same way, the hypothesis that independent subgroups of the unit group might be isomorphic to subgroups of $G$, conjecturally true in the case of the integral group ring of a finite group, was shown to be false for one of the groups of order 16 [14]. A further result for which these presentations have provided assistance is the following: for nonabelian $G$, the nilpotency class of the group of units assumes its

Received by the editor April 30, 1991.

1991 Mathematics Subject Classification. Primary 16S34; Secondary 20F05, 20D15, 20C05, 20C20, 16U60.

Key words and phrases. Modular group algebra, p-group, unit group, generators and relations, presentation of an extension. 
minimum value, $p$, if and only if the commutator subgroup $G^{\prime}$ is of order $p$ (the condition, equivalent to the vanishing of all $(p+1)$-fold Lie commutators, is sufficient as, for a $p$-group $G$ of nilpotency class 2 , the nilpotency class of $V(G)$ is less than or equal to the nilpotency class of the ideal $I\left(G^{\prime}\right)$ (cf. $[5,1]$ ); its necessity is shown in [11] (see also [17, 18])). Various other topics can be investigated by using presentations for small cases, for example, the problems with which [17] concludes.

A further reason lies in the history of the subject. The theory of modular representations of finite groups is a rich one but it has little to say about $p$ groups in characteristic $p$. For this case, the earliest papers $[22,8,21]$ remain the main sources of information. Moreover, the price of positive results often exceeds expectations: compare the very natural proof that an abelian group is determined by its integral group ring with the contrived one that a finite abelian $p$-group is determined by its modular group algebra (cf. [15]). The paucity of results suggests that theorems in this setting are few. It may be that the best that can be hoped for is a facility for answering questions about a specific group algebra.

The unit groups of modular group algebras of $p$-groups are encountered outside as well as within group theory. For example, they occur in algebraic $K$-theory in the context of localization (some references appear in [14]). Presentations for them were first obtained by Milnor (cf. [2, p. 602]) for cyclic $p$-groups. Specific questions in the case of dihedral and quaternion groups of order 16 were dealt with in an ad hoc manner in [20]. The construction given here provides a systematic manner of answering such questions. For the integral group ring, presentations for the unit group are sought in [4].

The group of normalized units in the case of a $p$-group is also a $p$-group. Structural features of a $p$-group can be calculated by machine, using software based on the Nilpotent Quotient Algorithm (NQA); CAYLEY [3], SOGOS [10], and GAP [13] are packages available publicly with this capacity. They apply to a $p$-group given in the form of a presentation by generators and relations. Thus, a unit group can be analyzed if a presentation of it is available.

Section 2 describes two algorithms for obtaining a presentation in this context. They function in an inductive manner grounded on the abelian $p$-group case [14] and are most practical when $p=2$, or when $p=3$ and the commutator subgroup is of order 3 . Section 3 lists those presentations obtained in this way which are small enough to convey in print, viz., for the groups of order dividing 16. The method has been successful in order 27 but the result is too complicated to include in a paper. With current NQA implementations, the method can be applied to groups of order 32 readily, order 64 probably, and order 81 possibly; improvements in, or alternatives to, NQA software could make larger orders accessible.

Throughout, $G$ will denote a finite $p$-group of order $|G|=p^{n}$ and $F$ will denote the field of $p$ elements, $I=I(G)$ denotes the augmentation ideal of the modular group algebra $F G$. As $I$ is the nilpotent radical of $F G, 1+I$ is a group; it is a $p$-group of order $p^{|G|-1}$ denoted by $V=V(G)$ and called the group of normalized units (since $U(F G)$, the group of all units of $F G$, is a direct product $U(F) \times V(G)$, it is only $V(G)$ that is at issue).

For a normal subgroup $N$ of $G$, the exact sequence 


$$
1 \rightarrow N \rightarrow G \rightarrow \bar{G} \rightarrow 1
$$

induces exact sequences of rings and of groups:

$$
\begin{aligned}
& 0 \rightarrow I(N) F G \rightarrow F G \rightarrow F \bar{G} \rightarrow 0, \\
& 1 \rightarrow 1+I(N) F G \rightarrow V(G) \rightarrow V(\bar{G}) \rightarrow 1 .
\end{aligned}
$$

Group commutators are denoted $[g, h]=g^{-1} h^{-1} g h$, and longer commutators are left-normed, e.g., $[x, a, b]=[[x, a], b]$. Repeated commutators are abbreviated by $[Q, k X]=[[Q,(k-1) X], X]$, where $[Q, X]$ denotes the set of all commutators $[q, x], q \in Q, x \in X$ (e.g., $[x, 3 a]=[x, a, a, a])$; $[Q, 0 X]$ denotes $Q$. The commutator subgroup of a group $H$ is denoted $H^{\prime}$, and its center by $\zeta(H)$.

The free group on a set $X$ of free generators is denoted by $F_{X}$. In a presentation $G \approx\langle X \mid R\rangle$, the elements of $R$ are strictly speaking relators so that $R \subseteq F_{X}$; nonetheless, they will be written and described sometimes as relators, sometimes as relations. The minimum number of generators of a group $H$ will be denoted $d(H)$; the minimum number of generators of an $F H$-module $M$ as module is denoted $d_{H}(M)$.

The cyclic group of order $n$ is denoted $C_{n}$ and the dihedral group of order $2 n$ is denoted $D_{n}$.

\section{THE METHODS}

As the elements of $V(G)$ are known explicitly and the multiplication of elements of $G$ is also known, the multiplication table of $V(G)$ is itself known. There is no more complete presentation for a group than its multiplication table! This presentation is of course impractical for the uses envisaged. More economical presentations can be found by putting together presentations for a nontrivial proper normal subgroup of $V(G)$ and for its quotient. This will always be done here in the setting in which the quotient is itself of the form $V(\bar{G}), \bar{G}$ a quotient of $G$, so that an inductive procedure can be developed.

Systematic approaches are given for two situations. That treated first applies to the special case of a nonabelian direct product. The general nonabelian case comes next. Presentations for abelian $p$-groups are obtained in an entirely theoretical manner in [14].

Case 1. Direct products. It is among the groups of order 16 that direct products involving nonabelian groups are first encountered. The structure of the unit group in such a case is easier to describe than in the general case, especially for $p=2$.

2.1. Proposition. Let $H$ and $K$ be finite p-groups, and let $G=H \times K$. Then $V(G)=(1+I(K) F H) V(H)$, a semidirect product.

If $p=2$ and $K \approx C_{2}$, then $1+I(K) F H \approx F H$ as right $F V(H)$-modules and $V(G) \approx F H \cdot V(H)$, where $F H$ is interpreted as an $F V(H)$-module by conjugation.

In such a case there is less need for a presentation of $V(G)$ as its decomposition as a semidirect product is often sufficient to provide any further information required about it. Nonetheless, for $p=2$, a presentation for $V\left(H \times C_{2}\right)$ 
is readily available from the decomposition. It is obtained by combining a presentation for $V(H)$ with a presentation for the elementary abelian group $F H$ and by indicating how $V(H)$ acts on $F H$.

2.2. Algorithm. A presentation for $V(G), G \approx H \times C_{2}, H$ a finite 2-group, may be obtained as follows:

Step 1. Presentation for $V(H)$.

This is assumed to be available by induction as $|H|<|G|$. Write $V(H) \approx$ $<X \mid R>$.

Step 2. Presentation for $F H$.

Choose a set $Y, Y \cap X=\varnothing$, in bijective correspondence with $H$. Then, in multiplicative notation, $F H \approx\langle Y \mid S\rangle$, where $S=\left\{\left[y, y^{\prime}\right], y^{2} \mid y, y^{\prime} \in Y\right.$, $\left.y \neq y^{\prime}\right\}$.

Remark. $Y$ is chosen in correspondence with a basis of $F H$. The relations in Step 3 are simplified by choosing the basis to contain a basis for $\zeta(F H)=$ $C_{F H}(H)=C_{F H}(V(H))$; recall that the class sums of $H$ give a basis for the center $\zeta(F H)$ of $F H$.

Step 3. Action of $V(H)$ on $F H$.

Recall that this is action by conjugation. For $y$ in $Y$ and $x$ in $X$, calculate the image in $F H$ of the word $[y, x]=y^{-1} y^{x}$. Then choose a word $u_{y, x}$ in the generators $Y$ which maps onto it. Write $T=\left\{[y, x] u_{y}^{-1} x \mid y \in Y, x \in X\right\}$.

Step 4. Presentation of $V\left(H \times C_{2}\right)$.

It is immediate from Proposition 2.1 that $V\left(H \times C_{2}\right) \approx\langle X, Y \mid R, S, T\rangle$.

Remark. For the practical implementation of this construction all that is needed are programs for carrying out calculations in $F H$ and $V(H)$. My programs are in Fortran and are based on reference to the multiplication table of $H$ obtained from CAYLEY or transcribed from [19].

This presentation is lengthy, owing to the large number of relations in $S$. It is possible to reduce the number of generators, but only at the expense of making the relations in $S$ and $T$ more complicated. Let $V=V\left(H \times C_{2}\right)$. Then $V / V^{\prime} \approx F H / F H \cdot I(V(H)) \times V(H) / V(H)^{\prime}$. This observation and the next lemma allow us to determine the minimal number of generators for $V$.

2.3. Lemma. Let $K$ be a finite p-group and $M$ a right $F K$-module with $I=$ $I(F K)$. Then $d_{K}(M)=\operatorname{dim}_{F}(M / M I)$.

2.4. Proposition. Let $H$ be a finite 2-group and $G=H \times C_{2}$. Then $d(V(G))=$ $d_{V(H)}(F H)+d(V(H))$, where $F H$ is interpreted as an $F V(H)$-module by conjugation.

Case 2. The general case. We now turn to the problem of presenting $V(G)$ in the general nonabelian case. Many aspects have close analogues in the special case. For example, the starting point is a central subgroup $Z$ of $G$ of order $p$ and its associated sequence

$$
1 \rightarrow 1+I(Z) F G \rightarrow V(G) \rightarrow V(\bar{G}) \rightarrow 1,
$$

where $\bar{G}=G / Z$ and an overbar is used to denote the projection. If presentations of $V(\bar{G})$ and $1+I(Z) F G$ are available, a presentation of $V(G)$ may be obtained by the construction of a presentation of an extension, as recorded in $[9$, pp. 138-140]. 
This is an approach well suited to induction. It can be assumed by induction that a presentation of $V(\bar{G})$ is available (based ultimately on a presentation for the unit group of an abelian $p$-group [14]). The group $1+I(Z) F G$ is known to an extent: it is, for example, of exponent $p$. For $p=2$ or 3 , more information is obtainable, and it is for these primes that the method given here is most practical.

2.5. Lemma. Let $G$ be a nonabelian finite p-group with central subgroup $Z$ of order $p$. Then $1+I(Z) F G$ is elementary abelian if and only if $p=2$, or $Z=G^{\prime}$ and $p=3$.

2.6. Algorithm. A presentation for $V(G), G$ a nonabelian $p$-group, may be obtained as follows:

Step 1. Choice of $Z$ and presentation of $V(\bar{G}), \bar{G}=G / Z$.

Choose $Z \leq G^{\prime} \cap \zeta(G),|Z|=p$. Define $\bar{G}=G / Z$. We assume by induction that a presentation $V(\bar{G}) \approx\langle X \mid R\rangle$ is available together with a presentation $\bar{G} \approx\left\langle X_{0} \mid R_{0}\right\rangle$ with $X_{0} \subseteq X$ and $R_{0} \subseteq R$. (The condition on $Z$ will enable us to obtain presentations for $G$ and $V(G)$ satisfying this property.) Write $F_{X}$ for the free group on $X$ and $\pi_{3}$ for the projection $F_{X} \rightarrow V(\bar{G})$ which gives the presentation $\langle X \mid R\rangle$.

Step 2. Presentation of $1+I(Z) F G: S$ relations.

Direct calculation of, for example, a power-commutator presentation is a practical undertaking in a sufficiently small case. We confine our discussion, however, to the case in which the hypotheses of Lemma 2.5 obtain. In this case, the subgroup $1+I(Z) F G$ is isomorphic to the vector space $I(Z) F G$. Calculation in the subgroup is then a matter of linear algebra in $F G$, particularly straightforward for $p=2$. My programs for this are in Fortran.

Fix a section $\sigma: V(\bar{G}) \rightarrow V(G)$ and define the homomorphism $\pi_{2}: F_{X} \rightarrow$ $V(G)$ by setting $\pi_{2}(x)=\sigma\left(\pi_{3}(x)\right)$ for each $x$ in $X$. Then $\pi_{2}\left(\langle R\rangle^{F_{X}}\right)=$ $\pi_{2}\left(F_{X}\right) \cap(1+I(Z) F G)$. In particular, $Z=\pi_{2}\left(\left\langle R_{0}\right\rangle^{F x_{0}}\right)=\pi_{2}\left(\left\langle R_{0}\right\rangle\right)^{G}$. Choose $r_{0}$ in $R_{0}$ such that $\pi_{2}\left(r_{0}\right)$ generates $Z$.

In $1+I(Z) F G$, calculate $\pi_{2}\left(r_{0}\right), \pi_{2}\left(R \backslash\left\{r_{0}\right\}\right)$, then $\pi_{2}([R, X]), \pi_{2}([R, 2 X])$, and so on; simultaneously, select a set of independent elements and express dependent elements of $\pi_{2}(R)$ in terms of them. Note that this process eventually ends with some $\pi_{2}([R, k X])=1$ because of the nilpotent action of $G$. Complete the independent set to a basis for $1+I(Z) F G$.

Let $Y_{1}$ and $Y_{2}, Y_{1} \cap Y_{2}=Y_{1} \cap X=Y_{2} \cap X=\varnothing$, be sets of free generators, $Y_{1}$ in bijective correspondence with the independent elements calculated above, and $Y_{2}$ in bijective correspondence with the remaining elements of the chosen basis. Put $Y=Y_{1} \cup Y_{2}$. Then $1+I(Z) F G$ has the presentation $\langle Y \mid S\rangle$, where $S=\left\{\left[y, y^{\prime}\right], y^{p} \mid y, y^{\prime} \in Y, y \neq y^{\prime}\right\}$. Thus, there are $m+\left(\begin{array}{c}m \\ 2\end{array}\right)$ relations of this type, where $m=\operatorname{rank}(1+I(Z) F G)=\frac{p-1}{p}|G|$. Write $F_{Y}$ for the free group generated by $Y$ and $\pi_{1}$ for the projection $F_{Y} \rightarrow 1+I(Z) F G$ which gives the presentation.

Step 3. Tietze transformations on $R$ and $S$.

In the previous step, a subset $R_{1}$ of $\bigcup\{[R, k X] \mid 0 \leq k<\infty\}$ has been made available which is in bijective correspondence with $Y_{1}$ and for which $\pi_{2}\left(R_{1}\right)$ is a basis of $\pi_{2}\left(F_{X}\right) \cap(1+I(Z) F G)$. By enlarging $R$, we will assume that $R_{1} \subseteq R$. 
For each $r$ in $R \backslash R_{1}, \pi_{2}(r)$ has been expressed as a linear combination of the elements of $\pi_{2}\left(R_{1}\right)$ in Step 2. Let $v_{r}$ be the corresponding product in the elements of $R_{1}$ so that $\pi_{2}\left(r v_{r}^{-1}\right)=1$. Write $\widetilde{R}=\left\{r v_{r}^{-1} \mid r \in R \backslash R_{1}\right\}$ with $\widetilde{R}_{0}$ similarly defined for $R_{0}$. Put $\widehat{R}=\widetilde{R} \cup\left\{r_{0}^{p}\right\} \cup\left\{\left[r_{0}, x_{0}\right] \mid x_{0} \in X_{0}\right\}$. The latter elements are adjoined in order that $G$ is presented as $\left\langle X_{0}\right| \widetilde{R}_{0} \cup\left\{r_{0}^{p}\right\} \cup$ $\left.\left\{\left[r_{0}, x_{0}\right] \mid x_{0} \in X_{0}\right\}\right\rangle$; this ensures that the condition imposed in Step 1 will obtain for any construction based on $G$.

Next use the elements in $R_{1}$ to replace appearances of elements of $Y_{1}$ in $S$, i.e., replace each $y$ in $Y_{1}$ by the element $r$ in $R_{1}$ corresponding to it. This results in a set $\widehat{S}$ of relations in the generators $X \cup Y_{2}$.

Step 4. Action of $V(G)$ on $1+I(Z) F G: T$ relations.

As in Algorithm 2.2, this action may be captured by specifying commutators. For each $y$ in $Y$ and $x$ in $X$, express $\left[\pi_{1}(y), \pi_{2}(x)\right]$ as a linear combination of the basis for $1+I(Z) F G$ defined in Step 2. Let $u_{y, x}$ be the corresponding product in the elements of $Y$. Put $T=\left\{[y, x] u_{y, x}^{-1} \mid y \in Y, x \in X\right\}$. Under the assumption that $|X|=d(V(\bar{G}))$, there are $m d(V(\bar{G}))$ such relations. Lastly, use Tietze transformations to replace each $y$ in $Y_{1}$ by the element $r$ in $R_{1}$ which corresponds to it. Again, this results in a set $\widehat{T}$ of relations in $X \cup Y_{2}$.

Step 5. Conclusion.

Collect the generating sets and relations from the previous steps to obtain the presentation $V(G) \approx\left\langle X, Y_{2} \mid \widehat{R}, \widehat{S}, \widehat{T}\right\rangle$.

This procedure can produce a great number of redundant relations. For example, it is common among the groups of order 16 for most of the relations $\widehat{S}$ to be consequences of the relations $\widehat{T}$. In contrast, the presentations given here in the tables have relatively few relations. Each has been reduced from that given by the general procedure by experimentation. A subset of relations can be established as redundant if, on its omission, the group so presented is of the same order. Calculation of orders was accomplished by use either of a coset enumeration implemented in CAYLEY, or of the Nilpotent Quotient Algorithm implemented in SOGOS.

Tietze transformations may be used to reduce the number of generators $Y_{2}$ by reference to the module structure of $1+I(Z) F G$ as in the discussion in the direct product case; it is a module by conjugation for the group $\bar{G}$ or even $\pi_{2}\left(F_{X}\right)$. These considerations prompt the following analogue to Proposition 2.4 .

2.7. Proposition. Let $Z$ be a central subgroup of order $p$ of the finite $p$-group $G$. Assume either that $p=2$ or that $p=3$ and $Z=G^{\prime}$. Let $\bar{G}=G / Z$, and let $W$ be the subgroup of $V(G)$ generated by the inverse images of a minimal set of generators of $V(\bar{G})$. Let $M_{1}$ denote the submodule $W \cap(1+I(Z) F G)$ of the $W$-module $M=1+I(Z) F G$ (via conjugation). Then $d(V(G)) \leq d_{W}\left(M / M_{1}\right)+$ $d(V(\bar{G}))$.

2.8. Illustration. The dihedral group $G$ of order 8 offers an illustration of the method of reasonable size. We sketch it here with less formality of notation. As $\zeta(G)=G^{\prime}$ is of order $2, Z=\zeta(G)$, so that $1+I(Z) F G$ is of rank 4 and $\bar{G}$ is elementary abelian of rank 2 . With $G=\left\langle x, y \mid x^{4}, y^{2},[x, y]=x^{2}\right\rangle$, we have $\bar{G}=$ $\left\langle\bar{x}, \bar{y} \mid \bar{x}^{2}, \bar{y}^{2},[\bar{x}, \bar{y}]\right\rangle$. From [14], $V(\bar{G})=\langle\bar{x}, \bar{y}, \bar{a}| \bar{x}^{2}, \bar{y}^{2}, \bar{a}^{2},[\bar{x}, \bar{y}],[\bar{a}, \bar{x}]$, 
$[\bar{a}, \bar{y}]\rangle$, where $\bar{a}=1+(\bar{x}-1)(\bar{y}-1)=\bar{x}+\bar{y}+\overline{x y}$. The subset $X$ of generators for $V(G)$ consists of the three elements $x, y$, and $a=x+y+x y$. The preimages of the relators are: $x^{2}, y^{2}=1, a^{2}=x+x^{2}+x^{3}+y+x y+x^{2} y+x^{3} y$, $[x, y]=x^{2},[a, x]=1+y+x y+x^{2} y+x^{3} y,[a, y]=1+x+x^{3}+x y+x^{3} y$. Of these, $x^{2}, a^{2},[a, x]$, and $[a, y]$ are independent. They form a basis of $1+I(Z) F G$, so that there are no generators $Y_{2}$ in this case and $d(V(G))=3$.

The relations $\widehat{R}$ are: $y^{2}=1,[x, y]=x^{2}, x^{4}=1,\left[x^{2}, x\right]=1,\left[x^{2}, y\right]=$ 1 . There are ten relations $\widehat{S}:\left[x^{2}, a^{2}\right]=1, x^{4}=1$, etc. There are 12 relations $\widehat{T}$, which turn out to express the fact that $1+I(Z) F G$ is central in $V(G)$; for example, $\left[a^{2}, x\right]=1$, so that $u_{a^{2}, x}$ may be taken $=1$. The six commutator relations in $\widehat{S}$ are clearly consequences of $\widehat{T}$. Further experimentation reduces the 27 relations of the general procedure to the ten which appear in the table for this group.

\section{THE GROUPS}

The constructions described in the previous section have been carried out for all the nonabelian groups of order dividing 16 and for the nonabelian group of order 27 and exponent 9 . The presentations obtained for the 2-groups are given in this section in tabular form. That obtained for the group of order 27 has not been included, it being too complicated for deductions by hand and too lengthy for manual transcription to machine. The minimal number of generators for it is 9, and 61 relations are used; this had been reduced from the 337 of Algorithm 2.6 by experimentation.

In contrast, the presentations obtained for the 2-groups are adequate for the purposes mentioned. As such, there was little reason to seek to reduce systematically all redundancy from the list of relations. Nonetheless, some effort has been expended in this direction and it is unlikely that much redundancy remains.

We begin by giving presentations in Table 1 (see next page) for all the nontrivial abelian 2-groups in the range. The information is merely that of [14] for these special cases. However, various of the groups of order 4 and 8 arise as the groups $\bar{G}$, so that the particular information is convenient here. The other groups appear for completeness.

Key for Table 1. Data sufficient for specifying a presentation of $V(G)$ for a given abelian group $G$ is given in a set of a few rows organized into columns. A set begins with the number of $G$ in the listing in [6] in the first column.

$G$ column: This gives the decomposition of $G$ as a direct product of cyclic groups.

$d(G)$ column: This gives the minimum number of generators of $G$.

Generators column: Standard names are used for the generators. $G=\left\langle g_{1}, \ldots\right.$, $\left.g_{d(G)}\right\rangle$, where $g_{1}=x, g_{2}=y, g_{3}=z$, and $g_{4}=w$. They are in the same order as that indicated in the $G$-column, i.e., $o\left(g_{1}\right) \geq o\left(g_{2}\right) \geq \cdots \geq o\left(g_{d(G)}\right)$. This information is given more explicitly in this column by listing each generator to the right of its order; thus, " $2: x, y$ " is to be interpreted as $o(x)=o(y)=2$.

$V(G)$ column: This gives the decomposition of $V(G)$ as a direct product of cyclic groups.

$d(V)$ column: This gives the minimum number of generators of $V(G)$. 


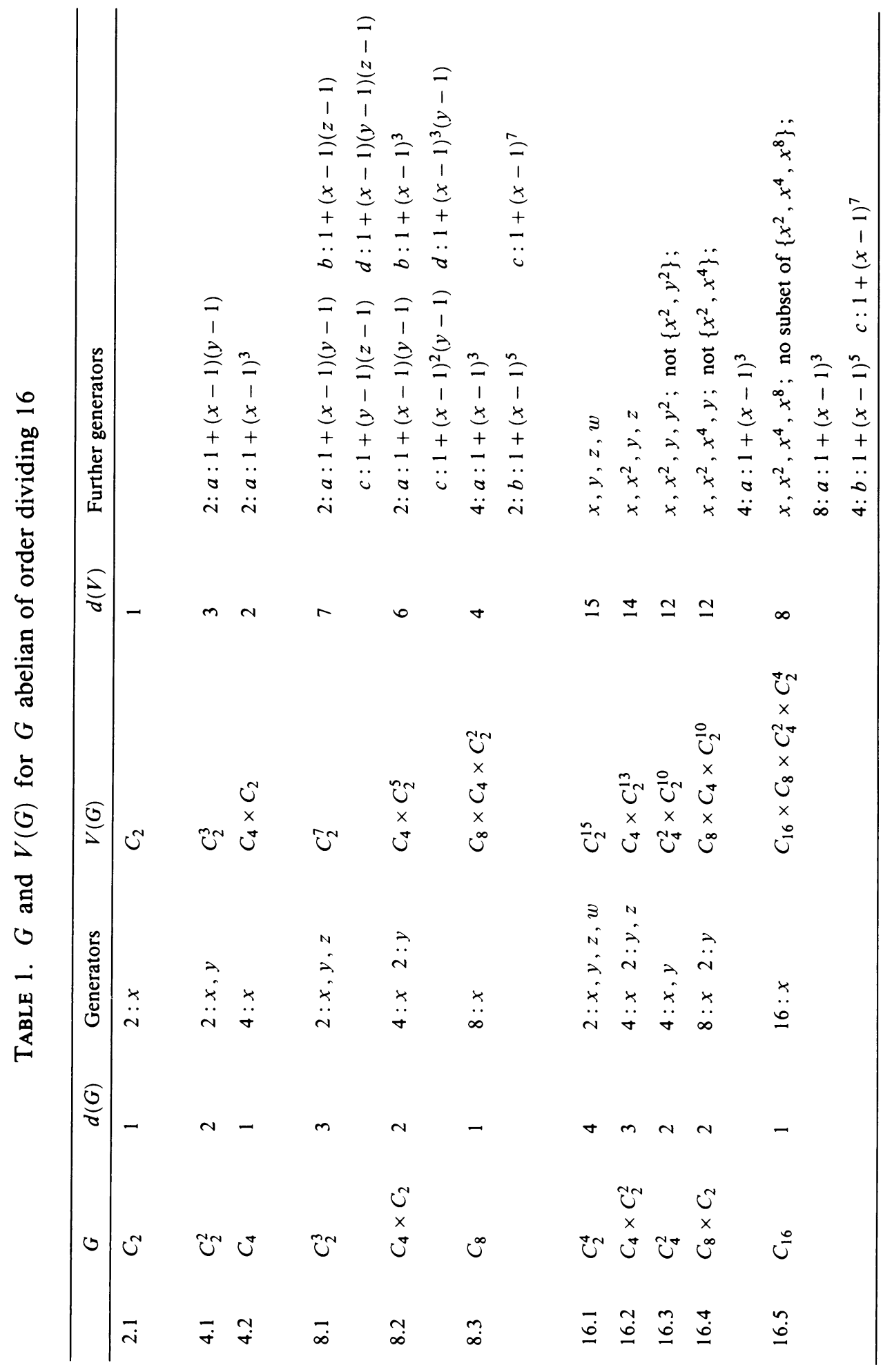


Further generators column: The generators of the factors in the decomposition of $V(G)$ are, first of all, the generators of $G$, and then those indicated in this column. The latter are given with their identification with group ring elements, the format for which is based on that of the Jennings basis rather than that of the group basis, " $a: 1+(x-1)(y-1)$ " means that the free generator $a$ maps onto the group ring element $1+(x-1)(y-1) \quad(=x+y+x y$ in characteristic 2) in the presentation for $V(G)$. The convention adopted in the fourth column gives the order of all named generators here. This data, together with the obvious commutator relations, which are omitted, specify the presentation of each $V(G),|G| \leq 8$. For groups of order 16 , the extra generators and power relations are not included in the table because of their length. They can be described in common via the Jennings basis: for each of these groups, there is a sequence of elements $x_{1}, x_{2}$, etc. such that the omitted generators correspond to elements among those of the form $1+\prod_{x \in Q}(x-1), Q \subseteq\left\{x_{1}, x_{2}, \ldots\right\}$, $|Q| \succcurlyeq 2$. For each case, the table gives three items of information: the sequence $x_{1}, x_{2}, \ldots$; the subsets $Q$ not to be used for generators; the elements corresponding to generators of order $\succcurlyeq 4$ (most subsets determine generators and most of these are of order 2). For example, for group 16.4, there is a generator of order 4 corresponding to the element $1+(x-1)\left(x^{2}-1\right)=1+(x-1)^{3}$; there is no generator corresponding to the subset $\left\{x^{2}, x^{4}\right\}$ but all the other subsets give generators or order 2, e.g., there is one for $\left\{x, x^{4}, y\right\}$ corresponding with $1+(x-1)^{5}(y-1)$.

Tables 2 and 3 describe $V(G)$ for $G$ nonabelian of orders 8 and 16. Again the code numbers of [6] are used for identification. Table 2 describes the groups $G$.

TABLE 2. Group descriptions

$8.4 \quad D_{4}$ dihedral

8.5 $Q$ quaternion

$16.6 \quad D_{4} \times C_{2}$

$16.7 Q \times C_{2}$

16.8 $\quad C_{4} \times C_{2}$ extended by $\left(\begin{array}{ll}1 & 0 \\ 2 & 1\end{array}\right)$

$16.9 \quad C_{4} \times C_{2}$ extended by $\left(\begin{array}{ll}1 & 1 \\ 0 & 1\end{array}\right)$

16.10 $C_{4}$ extended by an element of order 4 acting by inversion

16.11 $C_{8}$ extended by an involution centralizing $C_{4}$

$16.12 \quad D_{8}$ dihedral

16.13 $S D_{16}$ semidihedral

16.14 $Q_{16}$ generalized quaternion 


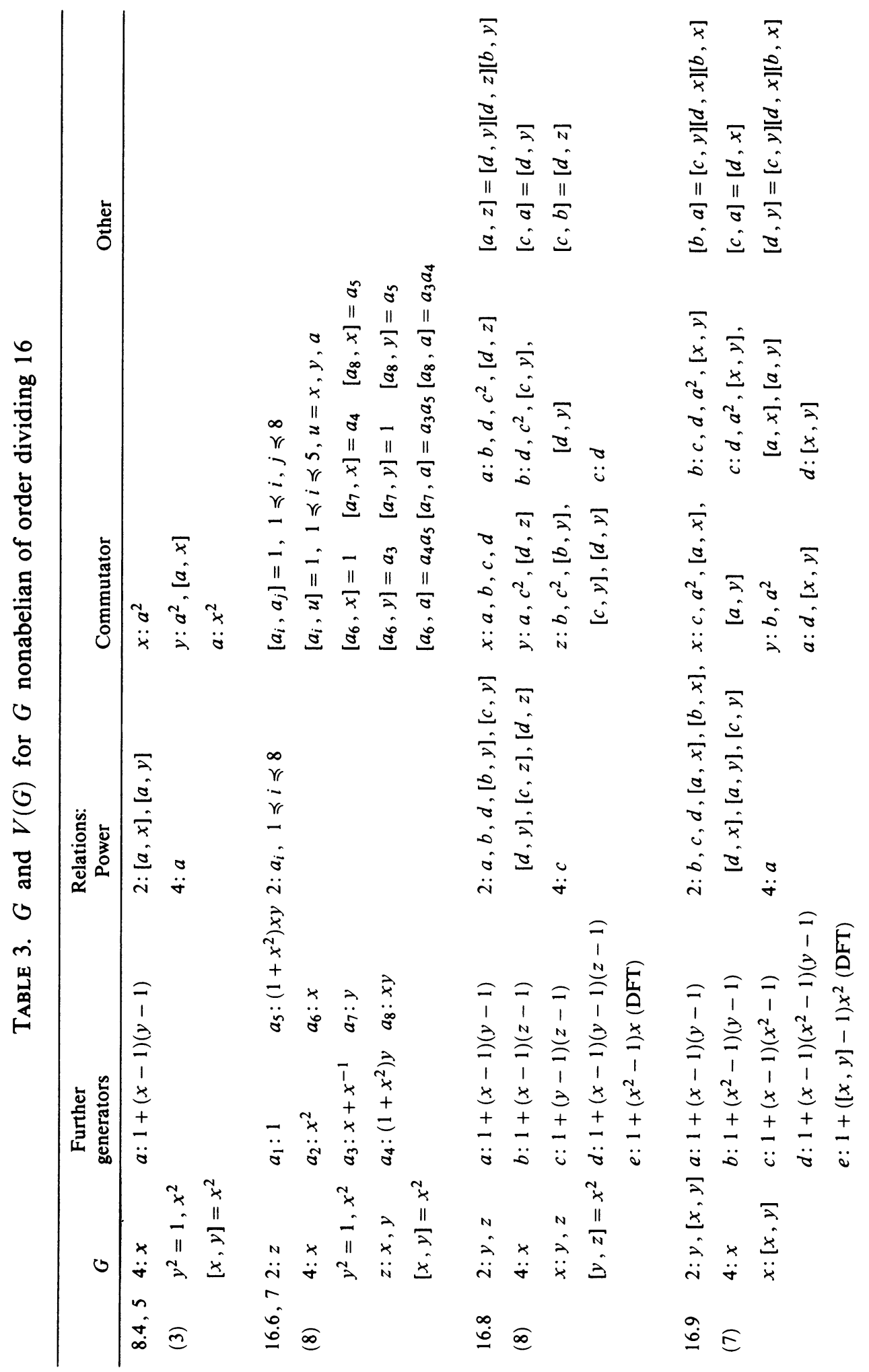




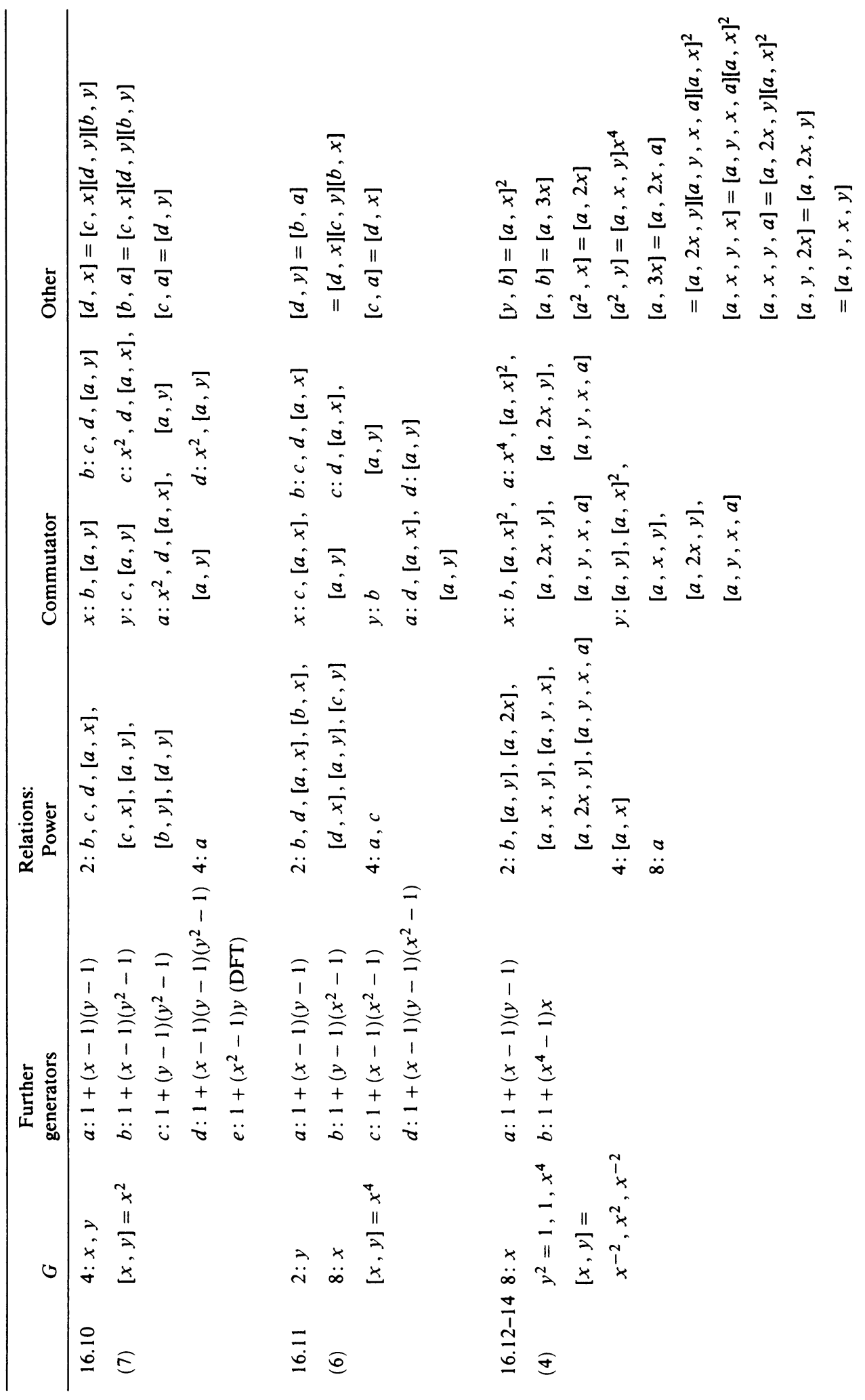


Table 3 (see pp. 698 and 699) indicates the presentations of $V(G)$ in a format analogous to that of Table 1. In several of the cases, the unit groups have presentations identical except for defining relations of the original group; in each of these cases, the data are merged into the same set of rows. For groups 16.6, 7, the presentation of Algorithm 2.2 is used so that the interpretation of the data here is somewhat different from that for the other groups.

Key for Table 3. The first column gives not only the code number of $G$ but also the minimum number of generators of $V(G)$ (in parentheses).

$G$ column: This gives a presentation for $G$ in free generators $x, y$, and, occasionally, $z$. As in Table 1, orders of elements are indicated by the notation: " $q: w_{1}, w_{2}$, etc.," meaning that $o\left(w_{i}\right)=q$. The fact that elements commute is also indicated by a shorthand: " $v: w_{1}, w_{2}$, etc.," meaning that $\left[w_{1}, v\right]=$ $1,\left[w_{2}, v\right]=1$, etc. In cases where data for different groups have been merged, the relevant variations in relations are given in order, separated by commas, e.g., in 8.4, $y^{2}=1$, while, in $8.5, y^{2}=x^{2}$.

Further generators column: For all but 16.6, 7, this column lists, one per row, the $k$ elements $v_{1}, \ldots, v_{k}$ of $V(G), k=d(V(G))-d(G)$, identified with free generators $f_{1}, \ldots, f_{k}$. Standard names are given to these generators: $f_{1}=a, f_{2}=b, f_{3}=c, f_{4}=d, f_{5}=e$. Together with the generators of $G, v_{1}, \ldots, v_{k}$ give a minimal generating set for $V(G)$. The last $d(G)-d(\bar{G})$ generators comprise $Y_{2}$. Such are present only in the cases 16.8-10 and in each case split off as a direct factor $\langle e\rangle$ of order 2 ; this is indicated in the table by the entry (DFT) after the element corresponding to $e$, and no further information about relations involving $e$ is given.

For $16.6,7$, generators $a_{1}, \ldots, a_{8}$ are given corresponding to a basis of $F H, H=\langle x, y\rangle$. These, together with the generators given in $8.4,5$ for $V(H)$, comprise the generating set used for $V(G)$; note that it is not minimal.

Power column: Relations of the form $w^{q}=1$ appear in this column according to the convention $q: w$ used earlier.

Commutator column: Relations of the form $\left[w_{1}, w_{2}\right]=1$ appear in this column according to the convention $w_{2}: w_{1}$ used earlier. For economy, this column is usually subdivided into two columns.

For $16.6,7$, the relations for $V(H)$ are given in the $8.4,5$ row of the table. All relations additional to those for $V(H)$ necessary for the presentation of $V(G)$ are given in this column; each gives the value of a commutator of two of the generators in the usual notation for a relation.

Other column: For all of the groups apart from $16.6,7$, the remaining relations for the presentation of $V(G)$ appear here in unabbreviated notation.

Illustration. For the dihedral group $D_{4}$, the table means that, for $D_{4} \approx\langle x, y|$ $\left.x^{4}=1=y^{2},[x, y]=x^{2}\right\rangle, V\left(D_{4}\right)$ has three generators $x, y, a$, corresponding to the group ring elements $x, y$, and $1+(x-1)(y-1)=x+y+x y$, and can be defined by ten relations in them. Its presentation is $V\left(D_{4}\right) \approx\langle x, y, a| x^{4}=$ $y^{2}=[a, x]^{2}=[a, y]^{2}=a^{4}=1,[x, y]=x^{2},\left[a^{2}, x\right]=\left[a^{2}, y\right]=[a, x, y]=$ $\left.\left[x^{2}, a\right]=1\right\rangle$. 


\section{ACKNOWLEDGMENTS}

I am grateful to Mike Newman and George Havas who first made available to me an implementation of the Nilpotent Quotient Algorithm and who instructed me in its use. I also thank John Cannon, Joachim Neubüser, and their teams for providing CAYLEY and SOGOS.

\section{BIBLIOGRAPHY}

1. C. Baginski, Groups of units of modular group algebras, Proc. Amer. Math. Soc. 101 (1987), 619-624.

2. H. Bass, Algebraic K-theory, Benjamin, New York, 1968.

3. J. J. Cannon, An introduction to the group theory language, Cayley, Computational Group Theory (M. D. Atkinson, ed.), Academic Press, London, 1984, pp. 145-183.

4. R. K. Dennis, The structure of the unit group of group rings, Ring Theory II (B. R. McDonald and R. A. Morris, eds.), Dekker, New York, 1977, pp. 103-130.

5. N. Gupta and F. Levin, On the Lie ideals of a ring, J. Algebra 81 (1983), 225-231.

6. M. Hall and J. Senior, The groups of order $2^{n} \quad(n \leq 6)$, Macmillan, New York, 1964.

7. L. R. Ivory, A note on normal complements in $\bmod p$ envelopes, Proc. Amer. Math. Soc. 79 (1980), 9-12.

8. S. A. Jennings, The structure of the group ring of a p-group over a modular field, Trans. Amer. Math. Soc. 50 (1941), 175-185.

9. D. L. Johnson, Presentations of groups, Cambridge Univ. Press, Cambridge, 1990.

10. R. Laue, J. Neubüser, and U. Schoenwaelder, Algorithms for finite soluble groups and the SOGOS system, Computational Group Theory (M. D. Atkinson, ed.), Academic Press, London, 1984, pp. 105-135.

11. A. Mann and A. Shalev, The nilpotency class of the unit group of a modular group algebra. II, Israel J. Math. 70 (1990), 267-277.

12. L. E. Moran and R. N. Tench, Normal complements in $\bmod$ p-envelopes, Israel J. Math. 27 (1977), 331-338.

13. A. Niemeyer, W. Nickel, and M. Schönert, GAP, Lehrstuhl D, Mathematik., Rhein.-Westf. Techn. Hochschule, Aachen, 1988.

14. R. Sandling, Units in the modular group algebra of a finite abelian p-group, J. Pure Appl. Algebra 33 (1984), 337-346.

15. _ The isomorphism problem for group rings: a survey, Orders and Their Applications (Oberwolfach, 1984), Lecture Notes in Math., vol. 1142, Springer, Berlin, 1985, pp. 256288.

16. _ The modular group algebra of a central-elementary-by-abelian p-group, Arch. Math. (Basel) 52 (1989), 22-27.

17. A. Shalev, On some conjectures concerning units in p-group algebras, Proc. 2nd Internat. Group Theory Conf. (Bressanone, 1989). Rend. Circ. Mat. Palermo (2) Suppl. 23 (1990), 279-288.

18. 70 (1990), 257-266.

19. A. D. Thomas and G. V. Wood, Group tables, Shiva, Nantwich, U.K., 1980.

20. C. T. C. Wall, Norms of units in group rings, Proc. London Math. Soc. (3) 29 (1974), 593-632.

21. H. N. Ward, Some results on the group algebra of a group over a prime field, Seminar on Finite Groups and Related Topics, Mimeographed notes, Harvard Univ., 1960-1961, pp. 13-19.

22. H. Zassenhaus, Ein Verfahren, jeder endlichen p-Gruppe einen Lie-Ring mit der Charakteristik p zuzuordnen, Abh. Math. Sem. Hansische Univ. 13 (1939), 200-207.

Department of Mathematics, The University, Manchester M13 9PL, England

E-mail address: rsandling@man.ac.uk 